\title{
Ecofriendly and Facile One-Pot Multicomponent Synthesis of Thiopyrimidines under Microwave Irradiation
}

\author{
Biswa Mohan Sahoo, ${ }^{1}$ B. V. V. Ravi Kumar, ${ }^{1}$ Jnyanaranjan Panda, ${ }^{1}$ and S. C. Dinda ${ }^{2}$ \\ ${ }^{1}$ Department of Medicinal Chemistry, Roland Institute of Pharmaceutical Sciences, Khodasingi, Berhampur 760 010, India \\ ${ }^{2}$ School of Pharmaceutical Education \& Research, Berhampur University, Berhampur 760 007, Odisha, India \\ Correspondence should be addressed to Biswa Mohan Sahoo; biswamohan81@gmail.com
}

Received 31 December 2012; Revised 20 February 2013; Accepted 25 February 2013

Academic Editor: Alexander Kauffmann

Copyright (c) 2013 Biswa Mohan Sahoo et al. This is an open access article distributed under the Creative Commons Attribution License, which permits unrestricted use, distribution, and reproduction in any medium, provided the original work is properly cited.

A rapid, improved, and ecofriendly synthesis of thiopyrimidines is carried out via one-pot multicomponent reaction of ethylcyanoacetate, substituted benzaldehydes, and thiourea in presence of ethanolic $\mathrm{K}_{2} \mathrm{CO}_{3}$ using microwave irradiation heating method. Excellent yields, shorter reaction time, and easy workup are the major advantageous features of this green protocol. So the application of multicomponent reactions involves the combination of multiple starting materials with different functional groups leading to the highly efficient and environmentally friendly construction of multifunctional drug molecules. The structures of the newly synthesized products were assigned on the basis of IR and ${ }^{1}$ HNMR spectral data.

\section{Introduction}

One-pot multicomponent organic reactions (MCORs) have emerged as an efficient tool for benign synthesis by virtue of their convergence, productivity, facile execution, and generation of highly diverse and complex products from easily available starting materials in a single operation. MCORs are now being tuned for synthesizing various heterocyclic compounds due to their diverse biological activities [1]. The environmental acceptability of the process is improved if the multicomponent strategy is applied under microwave irradiation (MWI) technology [2]. For an organic synthesis, a major adverse effect on the environment is the consumption of energy for heating. To overcome this problem, it is highly desirable to develop suitable methods of heating that use microwave irradiation. So, the synthetic methodologies nowadays should be designed in such a way to use and generate substances that possess little or no toxicity to human health and the environment. Microwave heating provides a valuable tool to perform reactions faster with enhanced product yields with high purity by reducing unwanted formation of byproducts [3]. From an environmental and economic perspective, it is becoming obvious that the traditional methods of performing chemical synthesis are unsustainable and have to be changed. Multicomponent coupling reactions provide a solution since they are more efficient, cost effective, and less wasteful than traditional methods. The achievement of making multiple bonds in a one-pot multicomponent coupling reaction promotes a sustainable synthetic approach to develop new drug molecule in a drug discovery process.

Microwave (MW) irradiation facilitates better thermal management of chemical reactions. The rapid MW heat transfer allows reactions to be carried out very much faster compared to conventional heating methods often resulting in increased product yield $[4,5]$. Furthermore, the products of temperature sensitive reactions from kinetic or thermodynamic pathways can be selectively tuned and isolated. The fundamental mechanism of microwave irradiated synthesis involves agitation of polar molecules or ions that oscillate under the effect of an oscillating electric or magnetic field. In the presence of an oscillating field, particles try to orient themselves or be in phase with the field. Only materials that absorb microwave radiation are relevant to microwave synthesis $[6,7]$.

These materials can be categorized according to the three main mechanisms of heating such as dipolar polarization, 
conduction, and interfacial polarization. In the electromagnetic spectrum, the microwave radiation region is located between infrared radiation and radiowaves. Microwaves have wavelengths of $1 \mathrm{~mm} \pm 1 \mathrm{~m}$, which corresponds to the frequencies between 0.3 and $300 \mathrm{GHz}$ [8, 9]. Microwave radiation is introduced into the reaction system remotely without direct physical contact with reaction materials, which can lead to a rapid increase in temperature throughout the sample causing less byproducts formation or decomposition of products. Microwave heating is able to heat the target compounds without heating the entire furnace or oil bath, which saves time and energy. In contrast, conventional heating of organic reactions such as oil baths, sand baths, or heating mantles is rather slow and creates an inward temperature gradient, which may result in localized overheating and reagent decomposition when heated for prolonged periods. In conventional heating method, the wall of reactor is heated by convection or conduction. The microwave-assisted organic synthesis has been classified into various categories such as microwave-assisted reactions using solvents, microwaveassisted reactions under solvent-free conditions, microwaveassisted reactions using solid-liquid phase, and microwaveassisted reactions on mineral supports in dry media $[10,11]$.

Since the multicomponent reactions often create the complete and complex molecular products in a single synthetic step, it is more accepted to describe this modern synthetic procedure as microwave multicomponent synthesis (MMS) $[12,13]$. The experimental benefits of generating complex structures from the simple starting materials without engaging protection-deprotection protocols and lengthy product purification procedures improve the synthetic approach for medicinal chemist wishing to contribute products to a more scientifically innovative society [14]. The previous facts prompted us to synthesize thiopyrimidines via onepot multicomponent reaction of ethylcyanoacetate, substituted benzaldehydes, and thiourea in presence of ethanolic $\mathrm{K}_{2} \mathrm{CO}_{3}$ using microwave irradiation heating method [1518]. Generally the one-pot multicomponent synthesis of thiopyrimidines follows the principle of Biginelli reaction protocol $[19,20]$.

\section{Experimental Method}

2.1. Materials. The melting points were taken in open capillaries and are uncorrected. The purity of the compounds was checked by TLC on precoated silica gel-aluminum plates and visualized by exposure to UV light $(254 \mathrm{~nm})$ or iodine vapor. The IR spectra of the compounds were recorded on FT-IR Spectrophotometer, IR Affinity-1 (SHIMADZU), using potassium bromide $(\mathrm{KBr})$ powder and the values are expressed in $\mathrm{cm}^{-1}$. ${ }^{1} \mathrm{H}$-NMR spectra of selected compounds were recorded on multinuclear FT-NMR Spectrometer, Advance-II (Bruker) (at $400 \mathrm{MHz}$ ), using tetramethylsilane $\left[\mathrm{Si}\left(\mathrm{CH}_{3}\right)_{4}\right]$ as an internal standard. The microwave irradiated synthesis was performed in scientific microwave oven (Catalyst System, Model No-CATA 2R). All the reactions were carried out at power level-2, which corresponds to $210 \mathrm{~W}$.
2.2. Chemistry. Multicomponent reactions (MCRs) constitute a highly valuable synthetic tool for the construction of polyfunctionalized heterocyclic compounds. Thus, a series of thiopyrimidine derivatives was synthesized based on multicomponent reaction (MCR) which involves one-pot organic reactions $[21,22]$. This reaction involves two mechanisms such as Knoevenagel condensation and Michael addition. First various substituted benzaldehydes $\left(\mathrm{RC}_{6} \mathrm{H}_{4} \mathrm{CHO}\right)$ react with ethylcyanoacetate $\left(\mathrm{NCCH}_{2} \mathrm{COOC}_{2} \mathrm{H}_{5}\right)$ in presence of ethanolic $\mathrm{K}_{2} \mathrm{CO}_{3}$ to produce an intermediate by Knoevenagel condensation reaction. Then the intermediate reacts with thiourea $\left[\mathrm{SC}\left(\mathrm{NH}_{2}\right)_{2}\right.$ ] via Michael addition to produce thiopyrimidines as depicted in Scheme 1.

\subsection{Synthesis of Thiopyrimidines}

2.3.1. Conventional Method. A mixture of appropriate substituted benzaldehydes $\left(\mathrm{RC}_{6} \mathrm{H}_{4} \mathrm{CHO}\right)$ (0.03 mole), ethylcyanoacetate $\left(\mathrm{NCCH}_{2} \mathrm{COOC}_{2} \mathrm{H}_{5}\right)(0.03$ mole, $3.39 \mathrm{~g})$, and thiourea $\left[\mathrm{SC}\left(\mathrm{NH}_{2}\right)_{2}\right](0.03$ mole, $2.28 \mathrm{~g})$ in ethanol $\left(\mathrm{C}_{2} \mathrm{H}_{5} \mathrm{OH}\right)(25 \mathrm{~mL})$ containing potassium carbonate $\left(\mathrm{K}_{2} \mathrm{CO}_{3}\right)(0.03$ mole, $4.15 \mathrm{~g}$ ) was taken and refluxed for $4-8 \mathrm{~h}$ on a water bath. The completion of reaction was monitored by TLC. The potassium salt of compound $4(\mathbf{a}-\mathbf{g})$, which precipitated during the reaction, was poured into cold water and acidified with glacial acetic acid $\left(\mathrm{CH}_{3} \mathrm{COOH}\right)$. The precipitate was filtered out, dried, and recrystallized from ethanol $\left(\mathrm{C}_{2} \mathrm{H}_{5} \mathrm{OH}\right)$.

2.3.2. Microwave-assisted Method. Required quantity of reactants and catalysts as mentioned previously was taken and refluxed under microwave irradiation for 5-10 min at power level-2 $(210 \mathrm{~W})$. The completion of reaction was monitored by TLC. The potassium salt of compound $\mathbf{4}(\mathbf{a}-\mathbf{g})$, which precipitated during the reaction, was poured into cold water and acidified with glacial acetic acid $\left(\mathrm{CH}_{3} \mathrm{COOH}\right)$. The precipitate was filtered out, dried, and recrystallized from ethanol.

\section{Results and Discussion}

In the present study, thiopyrimidine derivatives were synthesized by both conventional and microwave techniques. As compared to conventional method, microwave synthesis provides higher yield in lesser reaction time. From the results as summarized in Table 1, it can be observed that in case of conventional method of heating, the reactants are slowly activated by a conventional external heating source (water bath). Heat is driven into the reacting substances, passing first through the walls of the vessel in order to reach the solvent and the reactants. So, this is a slow method for transferring heat energy into the reaction medium. Hence, for the synthesis of the titled compounds of present work, which takes $4-8 \mathrm{~h}$ to complete the reaction. While considering microwave synthesis, microwaves couple directly with the reacting molecules of reaction mixture, leading to a rapid rise in the temperature. Since this process is not limited by the thermal conductivity of the vessel, the 


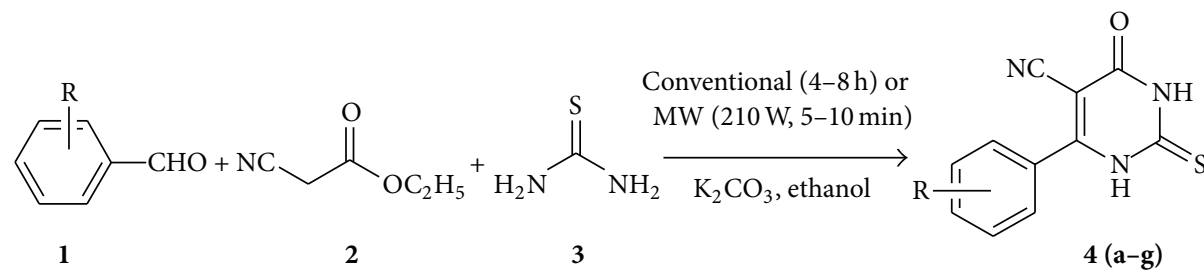

SCHEme 1: Synthetic route of the titled compounds $4(\mathbf{a}-\mathbf{g})$.

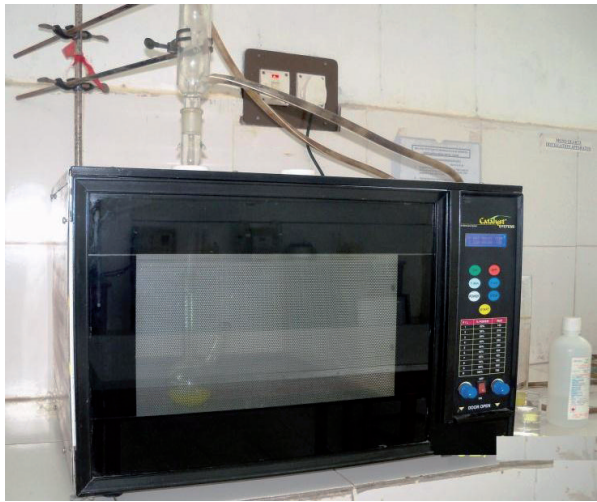

(a)

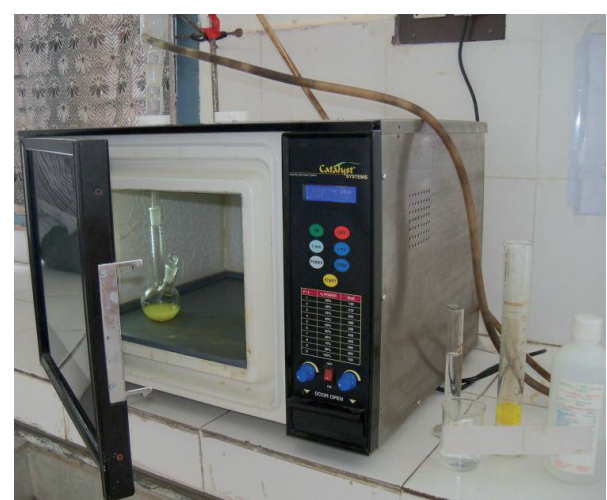

(b)

FIGURE 1: Photographs displaying microwave irradiated synthesis of titled compounds.

result is an instantaneous localized superheating of reacting substances that will respond to either dipole rotation or ionic conduction. Since the ability of a molecule to couple with the microwave radiation is a function of its molecular polarisability (i.e., a function of its dipole moment), only polar molecules interact with microwave energy. As a part of ongoing research of developing environmentally benign synthesis of thiopyrimidine derivatives, we explored a green multicomponent reaction (MCR) protocol using ethanol as solvent. Three component reactions such as the Biginelli reaction with ethylcyanoacetate, substituted benzaldehydes, and thiourea are well-known microwave-induced reactions (MW). Here an attempt was also made to carry out the synthesis of thiopyrimidine by microwave at power level-1 $(140 \mathrm{~W})$ but this energy was not sufficient to complete the reaction for getting final product. For the investigation of the optimized reaction condition, it was observed that the synthesis of target compounds by microwave required 5$10 \mathrm{~min}$ at power level-2 which corresponds to the energy of $210 \mathrm{~W}$. The photograph of microwave irradiated synthesis of titled compounds was given in Figure 1. During synthesis, thin layer chromatographic (TLC) study was carried out to monitor the completion of reaction using chloroform : ethyl acetate $(60: 40)$ as the mobile phase. The retention factor $\left(R_{f}\right)$ of the synthesized compounds was in the range of $0.32-0.51$. The melting points of the synthesized compounds were also checked and the results were given in Table 2. All the synthesized compounds are solid, white, crystalline, or amorphous. The photographs of some of the synthesized compounds were given in Figure 2. Most of the compounds are freely soluble in $\mathrm{CH}_{3} \mathrm{OH}, \mathrm{C}_{2} \mathrm{H}_{5} \mathrm{OH}, \mathrm{CH}_{2} \mathrm{Cl}_{2}$, $\left(\mathrm{CH}_{3}\right)_{2} \mathrm{SO},\left(\mathrm{CH}_{3}\right)_{2} \mathrm{NC}(\mathrm{O}) \mathrm{H}$. The compounds were analyzed by IR and ${ }^{1} \mathrm{H}-\mathrm{NMR}$ spectra. All the compounds showed an IR absorption band at a region $3410-3525 \mathrm{~cm}^{-1}$ which corresponds to the asymmetric stretching of $2^{0}-\mathrm{NH}$ group. They also showed the IR absorption band in the regions of $3150-3030 \mathrm{~cm}^{-1}, 2235-2198 \mathrm{~cm}^{-1}, 1795-1693 \mathrm{~cm}^{-1}, 1640-$ $1590 \mathrm{~cm}^{-1}, 1450-1530 \mathrm{~cm}^{-1}$, and $1380-1270 \mathrm{~cm}^{-1}$ for different groups like (Ar-H Str.), (CN Str.), ( $\mathrm{C}=\mathrm{O}$ Str.), ( $\mathrm{C}=\mathrm{C}$ Str.), (Sec. $\mathrm{N}-\mathrm{H}$ def.), and (C-N str.) vibration, respectively. The halogenated derivatives showed the IR absorption bands in the regions $900-1100 \mathrm{~cm}^{-1}, 755-780 \mathrm{~cm}^{-1}$, and $495-585 \mathrm{~cm}^{-1}$ corresponds to (C-F str.), and ( $\mathrm{C}-\mathrm{Cl}$ str.), (C-Br str.) vibrations respectively. The IR absorption band in the region 1230 $1255 \mathrm{~cm}^{-1}$ which corresponds to the $\mathrm{C}=\mathrm{S}$ str. vibration. The ${ }^{1} \mathrm{H}-\mathrm{NMR}$ spectra showed two singlets in the range $\delta 8.00-$ $8.30 \mathrm{ppm}$ due to $-\mathrm{NH}$ proton. They also showed the signals at $\delta 7.46-7.91 \mathrm{ppm}$ due to aromatic proton $(\mathrm{Ar}-\mathrm{H})$.

\section{Conclusion}

A new series of thiopyrimidine derivatives was synthesized successfully by both conventional and microwave irradiated methods to compare the reaction time and yield of the compounds. With the help of microwave synthesis, the yield of product increased from $55 \%$ up to $85 \%$ as compared to 


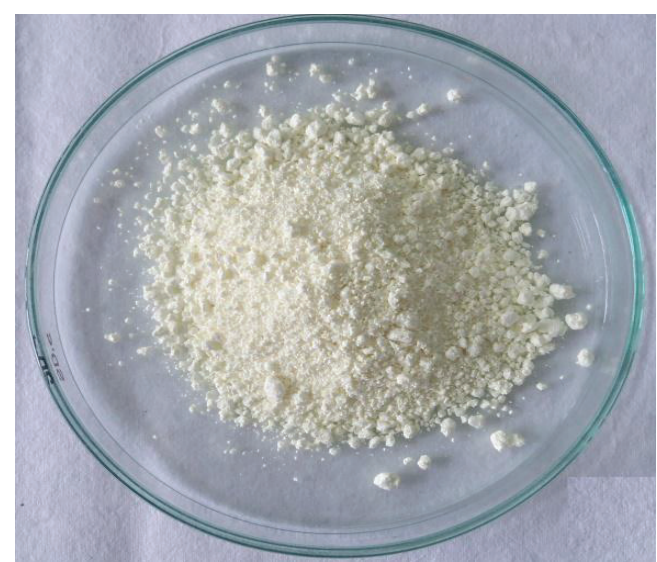

(a) Compound $\mathbf{4 a}$

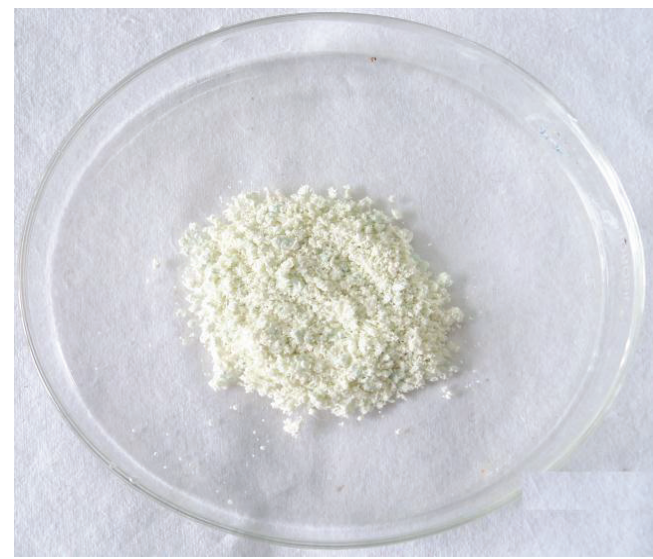

(c) Compound 4d

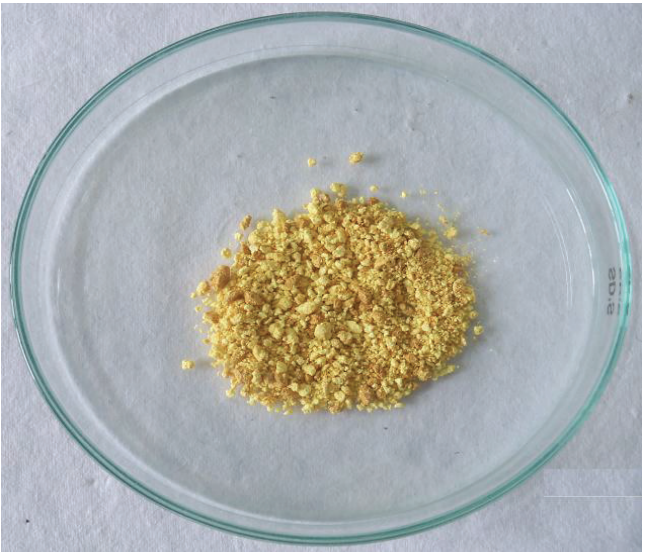

(e) Compound $\mathbf{4 f}$

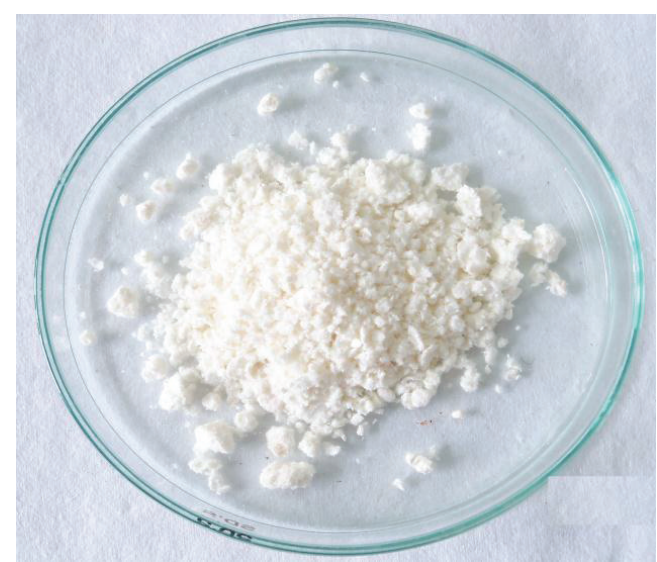

(b) Compound $\mathbf{4 b}$

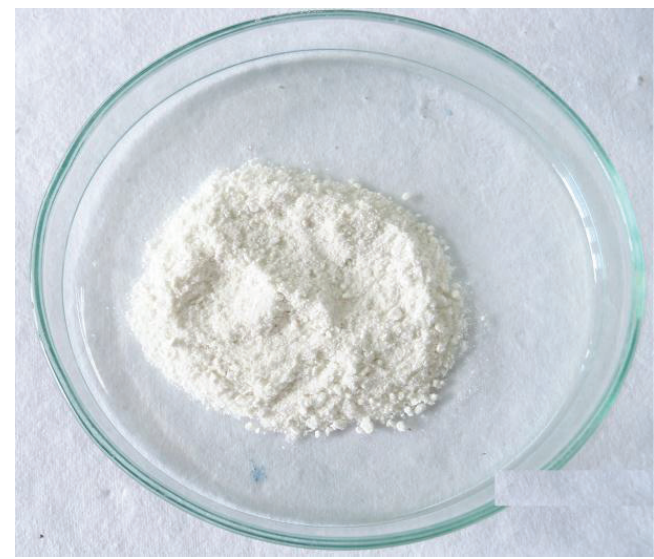

(d) Compound $4 \mathbf{e}$

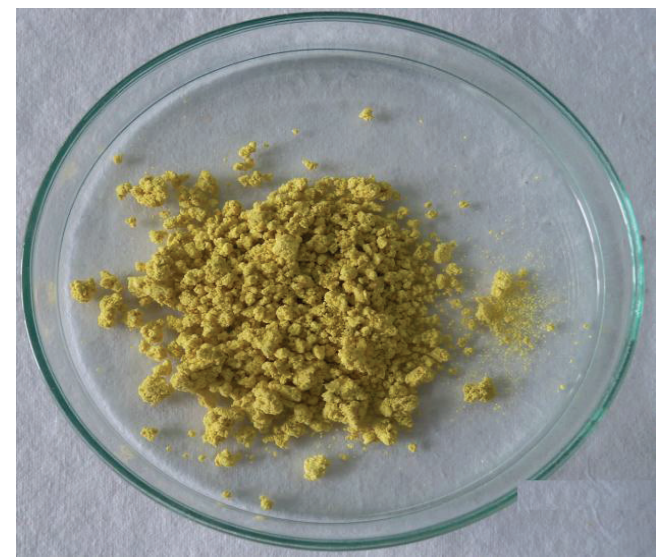

(f) Compound $4 \mathrm{~g}$

FIGURE 2: Photographs displaying some of the synthesized compounds by microwave irradiation method.

conventional synthesis. And also the reaction time is reduced from $4-8 \mathrm{~h}$ to $5-10 \mathrm{~min}$. Microwave synthesis reduced the formation of waste and byproduct. This is particularly relevant for high energy heterocyclic reactions. The predominant use of protic solvents leads to quicker, greener, and therefore more environmentally friendlyr reaction. The application of MW irradiation technology in MMS significantly enhanced the synthetic yield of thiopyrimidine obtained by consecutive Knoevenagel and Michael addition reactions performed on a mixture of substituted benzaldehydes, ethylcyanoacetate, and thiourea. MCRs have been proved as simple and convenient ways to produce a plethora of physiologically active thiopyrimidines. The strategy of MCR has been developed to enable the rapid construction of diverse structures from starting materials in a single operation. Multicomponent reactions are economically and environmentally very advantageous because multistep syntheses produce considerable amounts of waste mainly due to complex isolation procedures often 
TABLE 1: Comparative data on reaction time, heating energy, and yield of compounds $\mathbf{4}(\mathbf{a}-\mathbf{g})$ by conventional and microwave assisted synthesis.

\begin{tabular}{|c|c|c|c|c|c|c|}
\hline \multirow{2}{*}{ Compound code } & \multicolumn{3}{|c|}{ Conventional synthesis } & \multicolumn{3}{|c|}{ Microwave synthesis } \\
\hline & Time (h) & Energy (temp. $\left.{ }^{\circ} \mathrm{C}\right)$ & Yield (\%) & Time (min.) & Energy (power. watt) & Yield (\%) \\
\hline $4 a$ & 4 & $98-100$ & 66 & 5 & 210 & 85 \\
\hline $4 b$ & 6 & $98-100$ & 65 & 10 & 210 & 85 \\
\hline $4 c$ & 5 & $98-100$ & 62 & 7 & 210 & 82 \\
\hline $4 d$ & 8 & $98-100$ & 60 & 9 & 210 & 79 \\
\hline $4 e$ & 6 & $98-100$ & 61 & 6 & 210 & 82 \\
\hline $4 \mathrm{f}$ & 7 & $98-100$ & 55 & 8 & 210 & 78 \\
\hline $4 g$ & 6 & $98-100$ & 59 & 7 & 210 & 80 \\
\hline
\end{tabular}

TABLE 2: Melting point and TLC report of synthesized compounds $\mathbf{4}(\mathbf{a}-\mathbf{g})$.

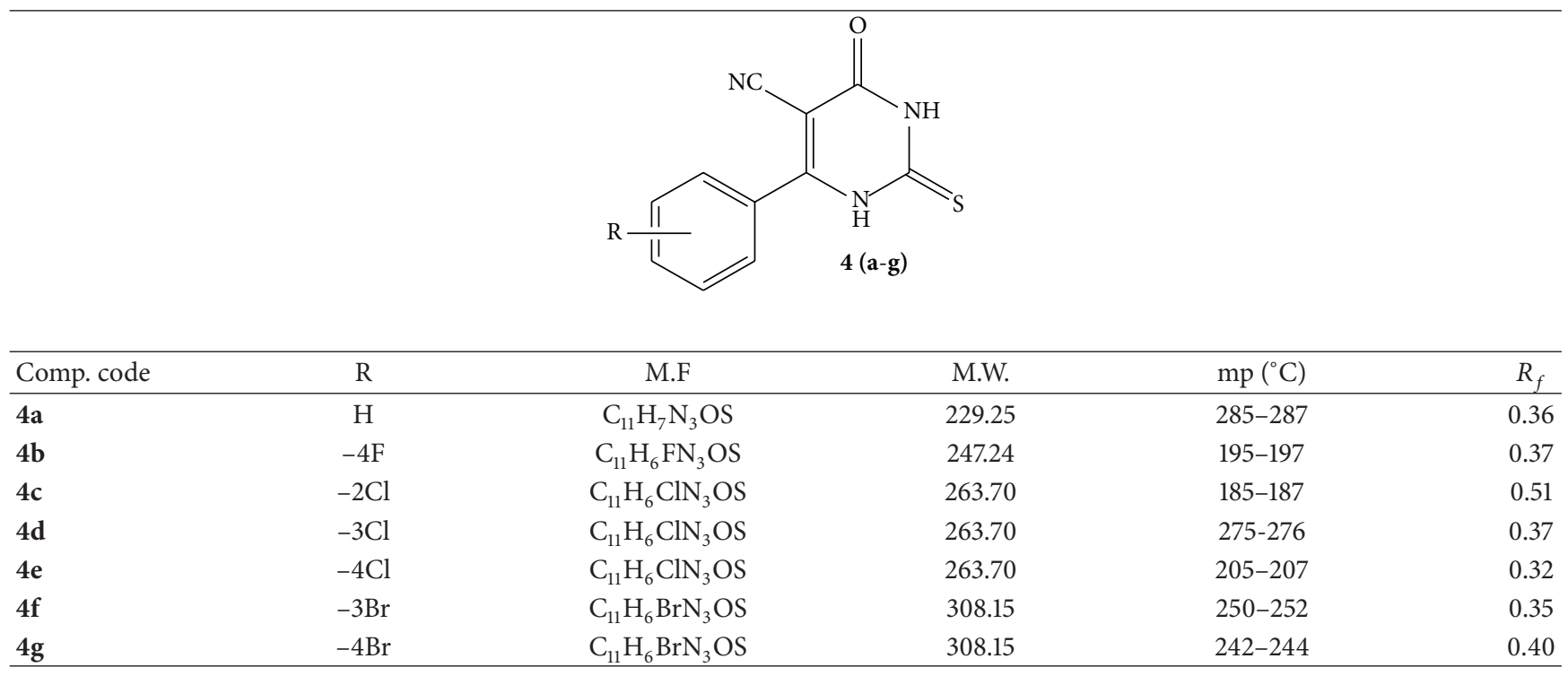

involving expensive, toxic, and hazardous solvents after each step. Thus, MCRs are suited for synthesis of compound libraries and are considered as an important tool during the developmental process of new drugs.

\section{Acknowledgments}

The authors are very much thankful to Roland Institute of Pharmaceutical Sciences, Berhampur, and School of Pharmaceutical Education \& Research, Berhampur University, Odisha, for providing necessary facilities to carry out the research work. The authors are also thankful to SAIF, Panjab University Chandigarh, for spectral analysis of synthesized compounds.

\section{References}

[1] M. C. Bagley and M. C. Lubinu, "Microwave-assisted multicomponent reactions for the synthesis of heterocycles," Journal of Heterocyclic Chemistry, vol. 1, pp. 31-58, 2006.

[2] S. Ravichandran and E. Karthikeyan, "Microwave synthesisa potential tool for green chemistry," International Journal of ChemTech Research, vol. 3, no. 1, pp. 466-470, 2011.
[3] M. Charde, A. Shukla, V. Bukhariya, J. Mehta, and R. Chakole, "A review on: a significance of microwave assist technique in green chemistry," International Journal of Phytopharmacy, vol. 2, no. 2, pp. 39-50, 2012.

[4] B. S. Sekhon, "Microwave-assisted pharmaceutical synthesis: an overview," International Journal of PharmTech Research, vol. 2, no. 1, pp. 827-833, 2010.

[5] M. A. Surati, S. Jauhari, and K. R. Desai, "A brief review: microwave assisted organic reaction," Archives of Applied Science Research, vol. 4, no. 1, pp. 645-661, 2012.

[6] P. Lidström, J. Tierney, B. Wathey, and J. Westman, "Microwave assisted organic synthesis-a review," Tetrahedron, vol. 57, no. 45, pp. 9225-9283, 2001.

[7] A. Loupy, Microwaves in Organic Synthesis, Wiley-VCH, Weinheim, Germany, 2006.

[8] C. O. Kappe, "Controlled microwave heating in modern organic synthesis," Angewandte Chemie International Edition, vol. 43, no. 46, pp. 6250-6284, 2004.

[9] F. R. Alexandre, L. Domon, S. Frère, A. Testard, V. Thiéry, and T. Besson, "Microwaves in drug discovery and multi-step synthesis," Molecular Diversity, vol. 7, no. 2-4, pp. 273-280, 2003.

[10] S. Caddick and R. Fitzmaurice, "Microwave enhanced synthesis," Tetrahedron, vol. 65, no. 17, pp. 3325-3355, 2009. 
[11] A. Madhvi, S. J. Surati, and K. R. Desai, "A brief review: microwave assisted organic reaction," Archives of Applied Science Research, vol. 4, no. 1, pp. 645-661, 2012.

[12] H. M. Hügel, "Microwave multicomponent synthesis," Molecules, vol. 14, no. 12, pp. 4936-4972, 2009.

[13] J. L. Krstenansky and L. Cotterill, "Recent advances in microwave-assisted organic syntheses," Current Opinion in Drug Discovery and Development, vol. 3, no. 4, pp. 454-461, 2000.

[14] M. Borisagar, K. Joshi, H. Ram, K. Vyas, and K. Nimavat, "A one-pot microwave irradiation synthesis of 1,2,4-triazolo[1,5A]pyrimidines," Acta Chimica \& Pharmaceutica Indica, vol. 2, no. 2, pp. 101-105, 2012.

[15] A. Kruithof, E. Ruijter, and V. A. Orru, "Microwave-assisted multicomponent synthesis of heterocycles," Current Organic Chemistry, vol. 15, no. 2, pp. 204-236, 2011.

[16] S. J. Vaghasia and V. H. Shah, "Microwave assisted synthesis and antimicrobial activity of some novel pyrimidine derivatives," Journal of the Serbian Chemical Society, vol. 72, no. 2, pp. 109117, 2007.

[17] J. M. Gajera and S. N. Tondlekar, "A novel and one-pot synthesis of 6-arylpyrimidin-4-ol," Research Letters in Organic Chemistry, pp. 1-3, 2008.

[18] N. Agarwal, P. Srivastava, S. K. Raghuwanshi et al., "Chloropyrimidines as a new class of antimicrobial agents," Bioorganic and Medicinal Chemistry, vol. 10, no. 4, pp. 869-874, 2002.

[19] K. Singh, K. Singh, B. Wan, S. Franzblau, K. Chibale, and J. Balzarini, "Facile transformation of Biginelli pyrimidin$2(1 \mathrm{H})$-ones to pyrimidines. In vitro evaluation as inhibitors of Mycobacterium tuberculosis and modulators of cytostatic activity," European Journal of Medicinal Chemistry, vol. 46, no. 6, pp. 2290-2294, 2011.

[20] C. O. Kappe, "A reexamination of the mechanism of the Biginelli dihydropyrimidine synthesis. Support for an N-acyliminium ion intermediate," Journal of Organic Chemistry, vol. 62, no. 21, pp. 7201-7204, 1997.

[21] M. Kidwai, K. Singhal, and S. Kukreja, "One-pot green synthesis for pyrimido[4,5-d]pyrimidine derivatives," Zeitschrift fur Naturforschung, vol. 62, no. 5, pp. 732-736, 2007.

[22] A. H. Kategaonkar, S. A. Sadaphal, K. F. Shelke, B. B. Shingate, and M. S. Shingare, "Microwave assisted synthesis of pyrimido[4,5-d]pyrimidine derivatives in dry media," Ukrainica Bioorganica Acta, vol. 1, pp. 3-7, 2009. 

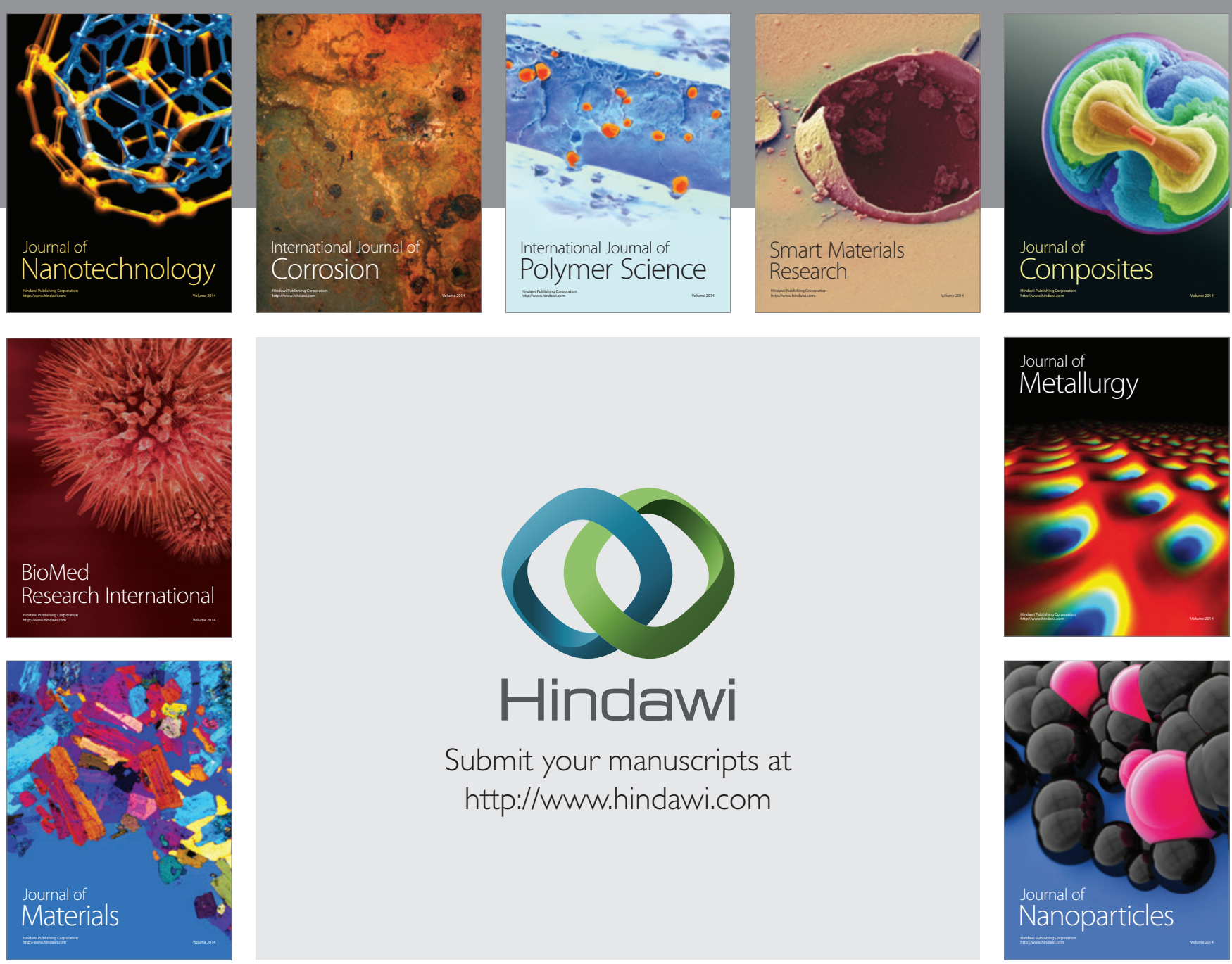

Submit your manuscripts at http://www.hindawi.com
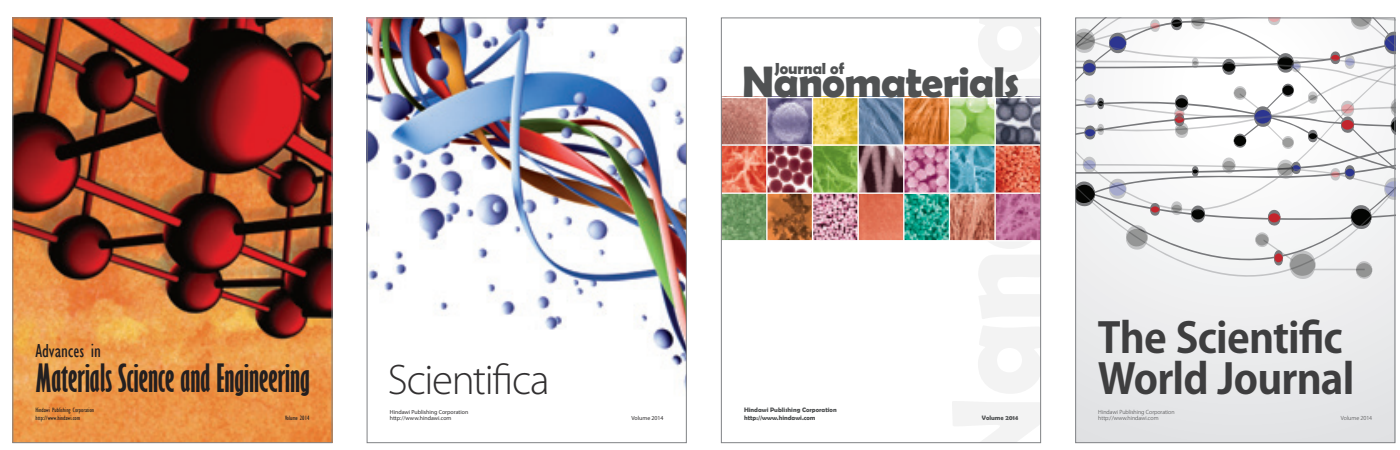

\section{The Scientific World Journal}
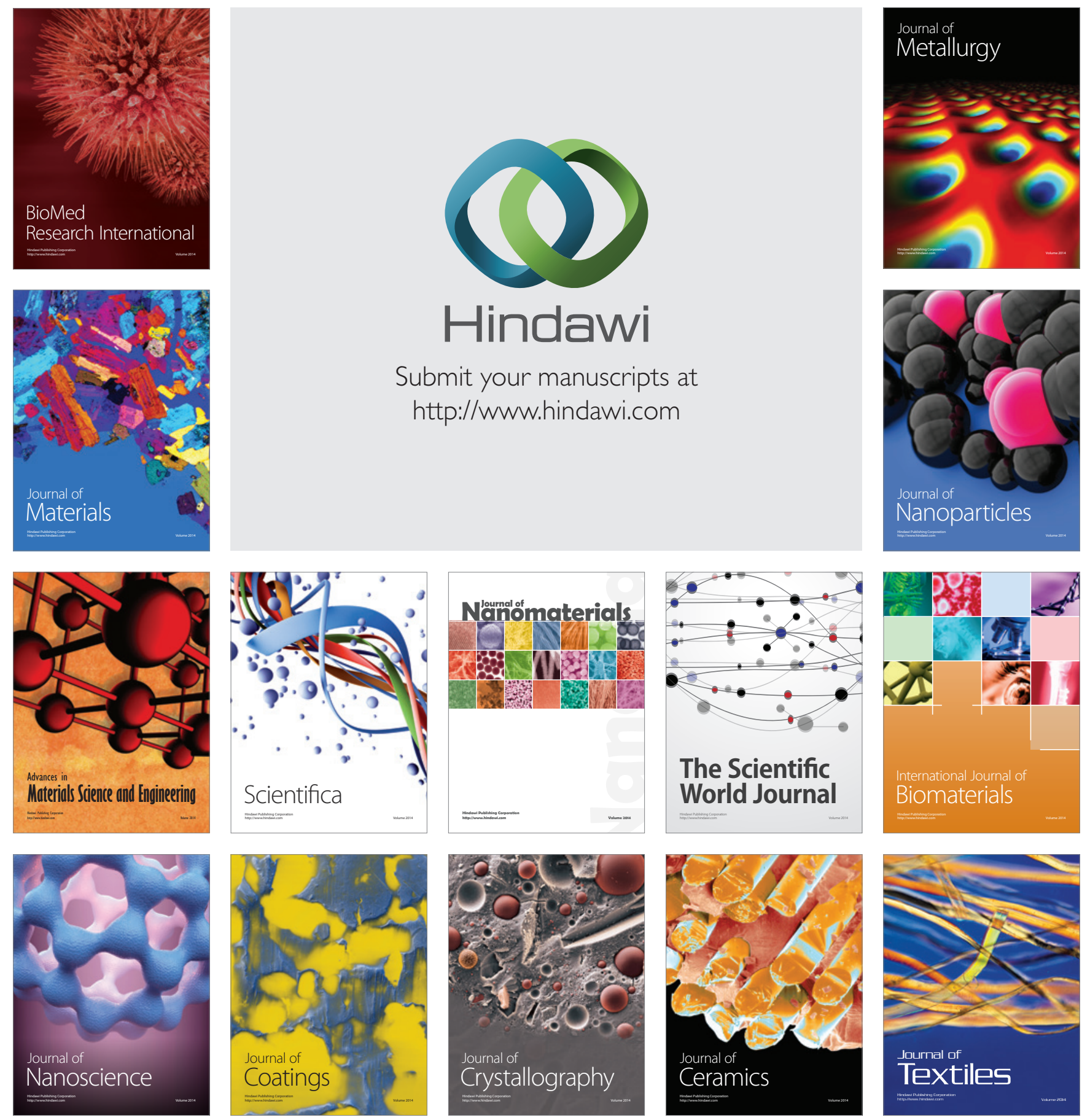